www.jmscr.igmpublication.org

Impact Factor (SJIF): 6.379

Index Copernicus Value: 71.58

ISSN (e)-2347-176x ISSN (p) 2455-0450

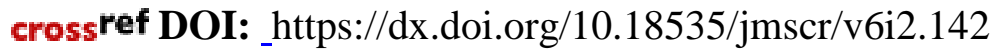

Journal Of Medical Science And Clinical Research

IGM Publication

An Official Publication of IGM Publication

\title{
Late Complication of Renal Transplantation Presenting As Lymphocele- Case Report and Literature Review
}

\author{
Authors \\ Dr Shahul Hameed ${ }^{1}$, Dr Avinash Naikwadi², Dr Rujuta Rege ${ }^{3}$ \\ ${ }^{1}$ [M.B.B.S, D.M.R.D] Head of the Dept of radiology at IQRA International Hospitals - Calicut, Kerala \\ ${ }^{2}$ [M.B.B.S, D.N.B] Consultant Department of radiology at IQRA International Hospitals - Calicut, Kerala \\ ${ }^{3}$ [M.B.B.S, M.D.] Consultant Department of radiology at IQRA International Hospitals - Calicut, Kerala \\ Corresponding Author \\ Dr Rujuta Rege
}

Address 1: 403/404 Richmond, Cross road no 2 , lokhandwala complex, andheri west, Mumbai - 400053. Address 2: Flat no 5 C, Skyline Palace Heights Appartment, Kottaram Road, Nadakkavu East, Calicut Kerala, Mob: 0091-9004453450

\section{Clinical Presentation}

A 26-year-old man who had undergone renal transplantation one year ago came for regular check -up. On examination his BP was $170 / 100 \mathrm{bpm}$ and pedal oedema was noted in bilateral lower limbs. Further investigation revealed a rising serum creatinine from 2.2 to 3.3 in a period of 4 days.

\section{Imaging Findings}

Ultrasound guided renal allograft biopsy was done to ascess the cause for the elevated serum creatinine. The histopathology report gave a diagnosis of hyaline thrombi with no evidence of graft rejection.

Ultrasound examination was done which revealed hydroureteronephrosis on the right (transplanted kidney) with a significant postvoid residue. $\{$ Fig $1\}$.

On catheterization $<50 \mathrm{ml}$ of urine was drained. Ultrasound examination was repeated and two fluid filled cavities were seen in the region of the pelvis. One was a anechoic peritransplant fluid collection seen causing hydroureteronephrosis and the second was the partially distended urinary bladder. \{Fig 2 and 3 \}.

\section{Treatment and Outcome}

Pigtail drainage of the peritransplant fluid was done under ultrasound guidance. $\{$ Fig $4 a$ and $4 b$ \} Subsequently there was reappearance of the collapsed/ partially distended bladder and decrease in the hydroureteronephrosis which was caused due to the compression of the ureters by the fluid filled cyst. $\{$ Fig 5$\}$.

Subsequently patients pedal oedema reduced and on repeat ultrasound of the abdomen there was resolution of the hydroureteronephrosis. 


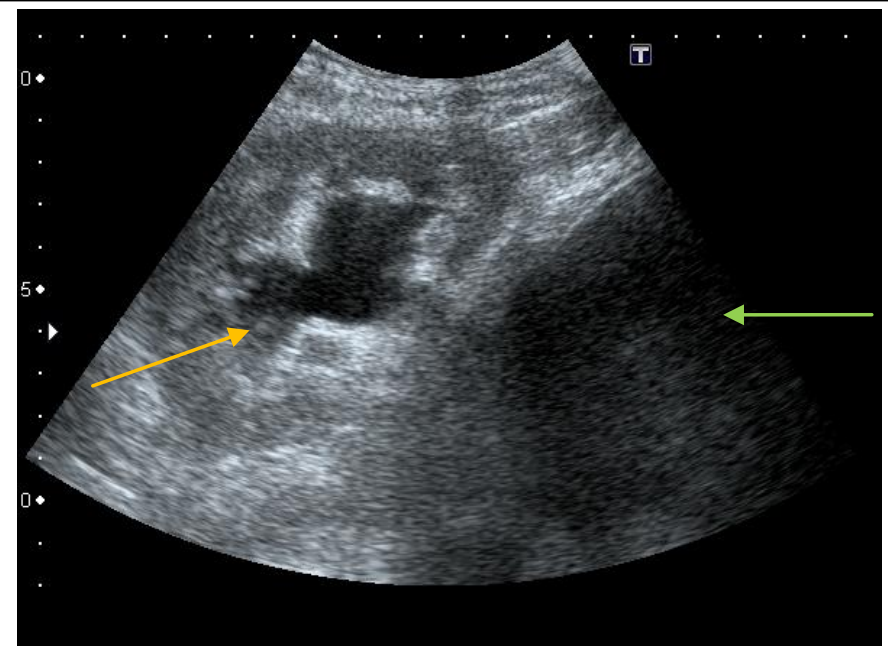

Fig 1: USG of the pelvis showing hydronephrosis (yellow arrow) and the perinephric lymphocele (green arrow)

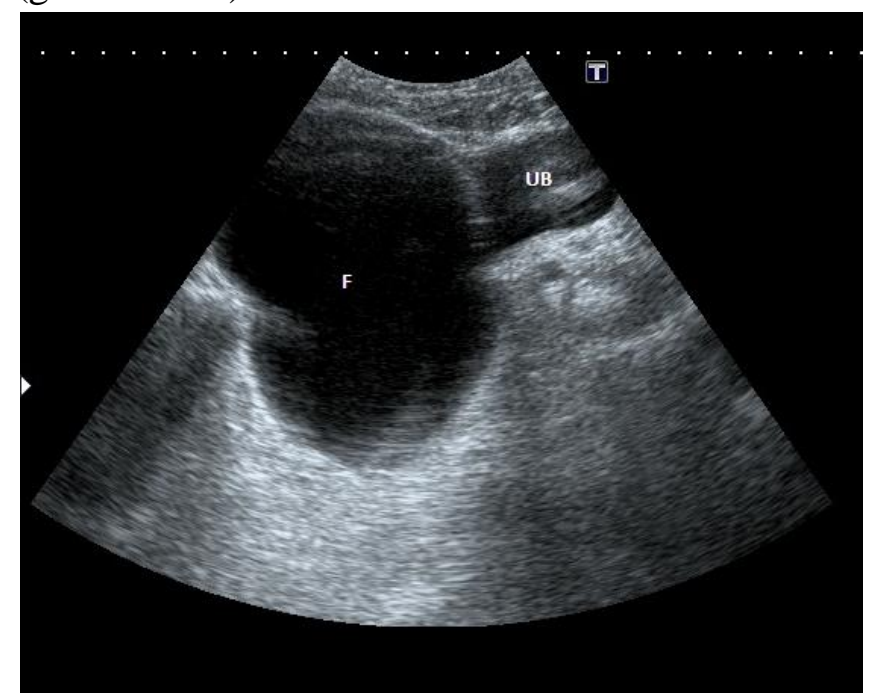

Fig 2: showing two anechoic fluid filed cavities in the region of the pelvis. The fluid marked with " $F$ " is the lymphocele whereas the UB is the urinary bladder.

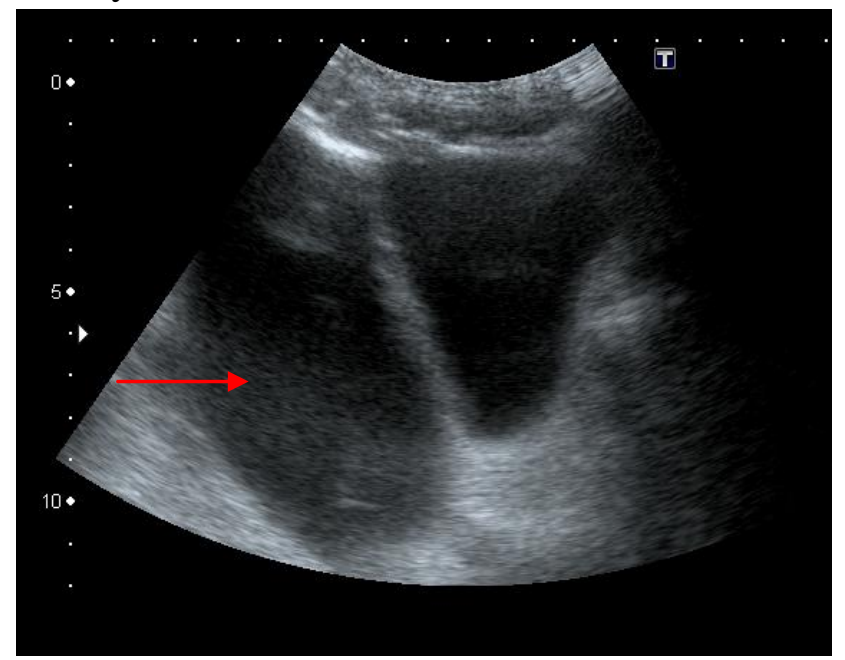

Fig 3: Two anechoic fluid filed cavities in the region of the pelvis. The fluid marked with red arrow is the lymphocele whereas the other is the urinary bladder.
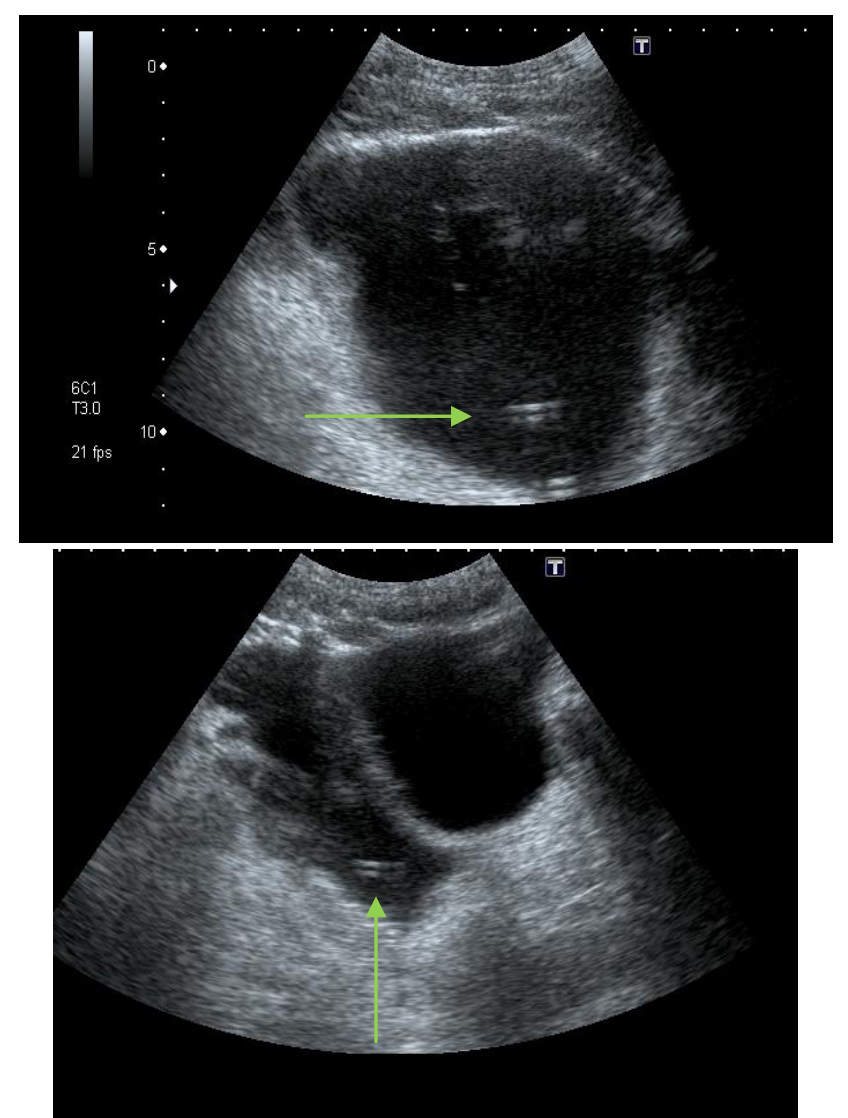

Fig 4 a and b: USG of the abdomen done showing the Pig tail catheter [green arrow] insitu within the lymphocele and the other fluid filled cavity is the urinary bladder. Note the decrease in size of the lymphocele soon after pigtail catheterization.

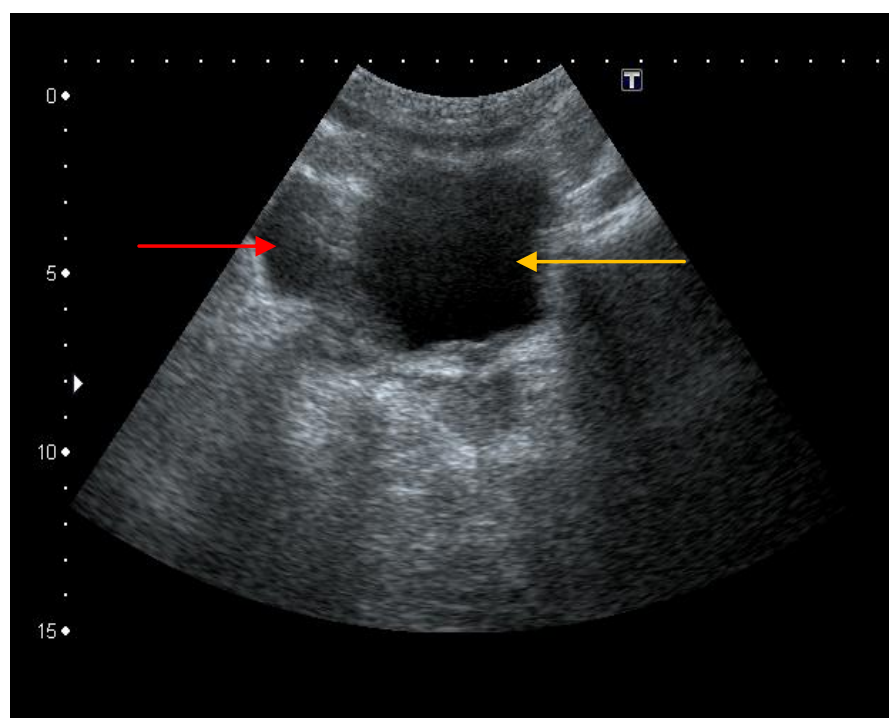

Fig 5: USG pelvis showing regressed lymphocele (red arrow) post pigtail catheterization and reappearance of the urinary ladder (yellow arrow). 


\section{Discussion}

Lymphoceles are the most common peritransplant fluid collections, with a prevalence of $0.5 \%-20 \%$. They may develop at any time, but are commonly seen in the early post operative period, upto few months after transplantation.

Lymphoceles are caused by leakage of lymph from surgically disrupted lymphatic channels along the iliac vessels or from the lymphatics of the transplanted kidney. Renal transplant patients are predisposed to prolonged lymphatic leakage as a result of graft rejection, the use of steroids or diuretics $^{(1)}$. Risk factors include inadequate ligation of the lymphatic channels across the iliac vessels, and possibly increased lymphatic flow secondary to edema of the lower extremities. These fluid collections usually occur medial to the transplant, between the graft and the bladder ${ }^{(2)}$.

Most lymphoceles are small and asymptomatic, and intervention is not necessary. However, some lymphoceles compress adjacent structures and may cause hydronephrosis, edema, or deep venous thrombosis in the ipsilateral lower extremity.

At US, lymphoceles are anechoic and may have septations. Similar to other peritransplant fluid collections, they can become infected and can develop a more complex appearance. At CT, lymphoceles have variable characteristics and are usually sharply circumscribed. Their CT attenuation values are typical of those of water and usually lower than those of recent hematomas and abscesses. Radionuclide and MR imaging studies are helpful for excluding the presence of urine and blood, respectively. Small lymphoceles are monitored sonographically, and large ones, if they grow or cause hydronephrosis, should be drained. Aspirated fluid should have a creatinine content equal to that of serum ${ }^{(3)}$.

Lymphoceles commonly recur after simple percutaneous or surgical drainage. Permanent resolution of lymphoceles may require prolonged catheter drainage and transcatheter instillation of sclerosing agents such as povidine-iodine, absolute alcohol, or doxycycline ${ }^{(3)}$. Repeated aspirations run the risk of infecting an otherwise sterile fluid collection. However, success rates of up to $97 \%$ have been achieved safely and effectively with percutaneous transcatheter sclerotherapy ${ }^{(4)}$.

\section{Conclusion}

Transplantation is one of the established treatments of irreversible kidney disease. Improvements in surgical techniques and more sophisticated, potent immunosuppressive drugs have resulted in remarkable advances in survival of patients and renal grafts. Nevertheless, substantial complications occur in both the immediate postoperative period and later. Imaging has a critical role in the evaluation of these complications, and interventional radiologic techniques are often successfully applied to their treatment.

Ultrasound-guided drainage with a success rate greater than $50 \%$ should be recommended as the first line of treatment. As a minimal invasive surgery this kind of treatment does not interfere with subsequent internal drainage through an open or a laparoscopic surgery. Laparoscopy, a feasible, safe technique with a success rate of more than $80 \%$, should be used routinely after unsuccessful percutaneous drainage.

\section{References}

1. Complications of Renal Transplantation (2005) Syed A. Akbar, MD , S. Zafar H. Jafri, MD, Marco A. Amendola, MD et al. Radio Graphics 2005; 25:1335-1356.

2. Khauli RB, Stoff JS, Lovewell, et al. Post -transplant lymphoceles: A critical look into the risk factors, pathophysiology and management. JUrol1993; 150: 22-26.

3. Brockis JG, Hulbert JC, Patel AS, et al. The diagnosis and treatment of lymphoceles associated with renal transplantation: a report of 6 cases and review of the literature. Br J Urol1978; 50: 307-312.

4. Van Sonnenberg E, Wittich GR, Casola G, et al.Lymphoceles: Imaging characteristics 
and percutaneous management. Radiology 1986; 161: 593-596.

5. Montalvo BM, Yrizarry JM, Casillas VJ, etal. Percutaneous sclerotherapy of lymphoceles related to renal transplantation. J Vas Intery Radiol 1996; $7: 117-123$. 